\title{
The Design and Implementation of WiMAX Module for ns-2 Simulator
}

\author{
Frank Chee-Da Tsai ${ }^{\star}$, Jenhui Chen ${ }^{\dagger}$, Chiang-Wei Chang, Wei-Jen Lien, Chih-Hsin Hung, and Jui-Hsiang Sum \\ ${ }^{\star}$ Networks and Multimedia Institute, Institute for Information Industry \\ $\dagger$ Department of Computer Science and Information Engineering \\ Chang Gung University, Kweishan, Taoyuan, Taiwan, R.O.C.
}

\begin{abstract}
Currently the network simulator 2 (ns-2) is a popular and powerful simulation for IEEE 802.11 wireless networks, including wireless LANs, mobile ad hoc networks (MANETs), and sensor networks. However, the network module has not been extended to IEEE 802.16 broadband wireless access networks (BWANs) or WiMAX yet. In this paper, we design and implement a new medium access control (MAC) protocol based on the IEEE 802.16 standard with the point-to-multipoint (PMP) mode for the ns-2. This module will benefit academic researchers and industrial developers in developing the WiMAX system. Besides, the architecture of the module is also presented in this paper.
\end{abstract}

Index Terms-MAC, module, simulator, WiMAX.

\section{INTRODUCTION}

Broadband wireless access (BWA) has received much more attentions in recent years [6], [7], [10]. Research in the area of wireless networks increasing as the number of wireless network devices will soon surpass the number of wired network devices. Fixed BWA systems, such as the local multipoint distribution service (LMDS), provide multimedia services to a number of discrete subscriber sites with IP and offer numerous advantages over wired IP networks. This is accomplished by using base stations (BSs) to provide network access services to subscriber sites based on the IEEE 802.16 WirelessMAN standard. First published in April 2002, IEEE 802.16 standard has recently been updated to IEEE 802.162004 [1] (approved in June 2004). The standard focuses on the "first-mile/last-mile" connection in wireless metropolitan area networks (WMANs) [5].

Its purpose is to facilitate the optimal use of bandwidth 2-66 $\mathrm{GHz}$, as well as interoperability among devices from different vendors. Typical channel bandwidth allocations are 20 or 25 $\mathrm{MHz}$ (United Stats) or $28 \mathrm{MHz}$ (Europe) in $10-66 \mathrm{GHz}$, or various channel bandwidths among 1 to $30 \mathrm{MHz}$ in $2-11 \mathrm{GHz}$ [2]. The progress of the standard has been fostered by the keen interest of the wireless broadband industry to capture the emerging WiMAX (worldwide interoperability for microwave access) market, the next-wave wireless market that aims to provide wireless broadband access services. IEEE 802.16 is the current trends on WiMAX. The WiMAX Forum, formed in 2003, is promoting the commercialization of IEEE 802.16 and the European Telecommunications Standard Institute's

\footnotetext{
${ }^{\dagger}$ Corresponding Author: Prof. J. Chen, Department of Computer Science and Information Engineering, Chang Gung University, Kweishan, Taoyuan, Taiwan. Email: jhchen@mail.cgu.edu.tw. This work was supported by the Innovative and Prospective Technologies Project of Institute for Information Industry (III) and sponsored by MOEA, Taiwan, R.O.C.
}

(ETSI's) high performance radio MANs (HyperMANs). It provides one of potential solutions to $\mathrm{B} 3 \mathrm{G} / 4 \mathrm{G}$ architecture.

The most popular network simulator used by the academia and industry is the network simulator 2 (ns-2) [11], which has become the de facto standard for the simulation of packet-switched networks. Specifically, more and more published wireless network studies and investigations use ns-2 to evaluate and verify their work. Although there still another force investigates the IEEE 802.16-based simulator [8], this simulator is not public. The ns-2 is roughly composed of various traffic models, transport-layer protocols, network-layer protocols, and medium access control (MAC) layer protocols, etc. These components enable ns-2 to simulate different types of networks and their topologies. Researchers can benefit from these preliminary tests on their investigation and find out the drawbacks of their new design in efficient way.

Until today, as the best knowledge of the authors, no WiMAX/IEEE 802.16 module has been contributed to ns-2. Due to these reasons, we design and implement the WiMAX module for ns-2. The developed WiMAX module is focused on MAC protocol development and inherited from the original MAC class in ns-2. This module is based on IEEE 802.16 point-to-multipoint (PMP) mode, which means that one BS can serve multiple subscriber stations (SSs) concurrently. We choose the orthogonal frequency-division multiple access (OFDMA) scheme for the physical (PHY) layer. Based on the OFDMA PHY specifications, it has been of major interest for both wireless applications due to its high date rate transmission capability and its robustness to multipath delay spread [9].

The IEEE 802.16 standard defines the specifications related to the service-specific convergence sublayer (CS), the MAC common part sublayer (CPS), the security sublayer, and the PHY layer. The MAC management messages, such as downlink/uplink map (DL-MAP/UL-MAP), downlink/uplink channel descriptor (DCD/UCD), ranging request/response (RNGREQ/RNG-RSP), and so forth are implemented following the 802.16 standard to operate the WiMAX networks. All operations between the base station (BS) and subscriber stations (SSs) over a superframe interval follow the compulsory procedures of the 802.16 standard. A detailed IEEE 802.16 based WiMAX module can be found in following sections.

In addition, we adopt the unified modeling language (UML) to design and analyze the developed WiMAX module in the early stage. The UML allows programmers to easily get the visualization and analysis of the required system models in the WiMAX module. The UML is also applicable to the context 


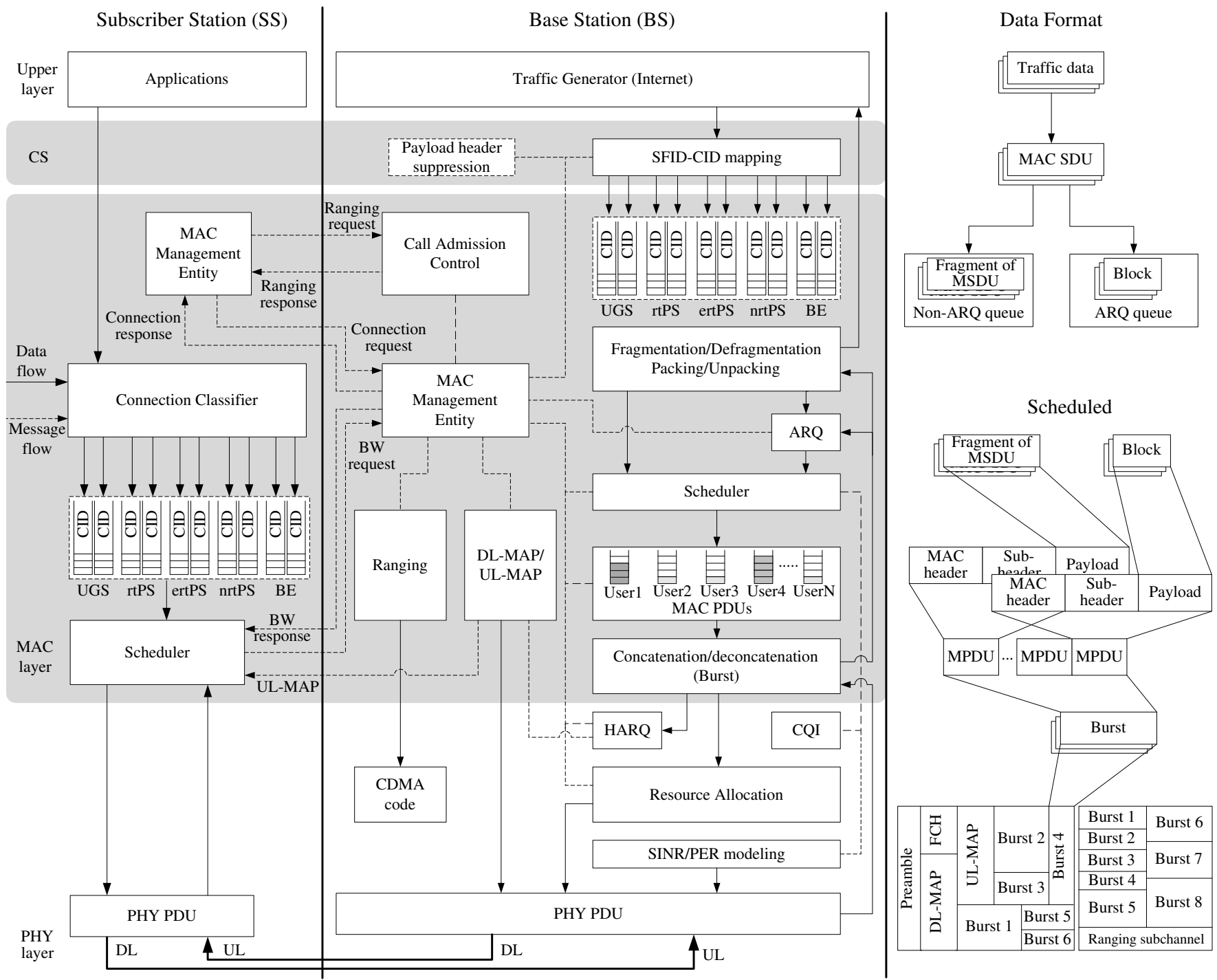

Fig. 1. The MAC simulation architecture of IEEE 802.16.

of a paradigm that enhances the capability of modeling the program and simplifies the management and maintenance of the module.

The remainder of this paper is organized as follows. Section II takes an overview of the IEEE 802.16 architecture. Section III describes all classes built in the module in detail. In Section IV, we demonstrate the usage of the WiMAX module and its related simulation parameters in detail. Finally, some conclusions are discussed in Section V.

\section{AN OVERVIEW OF THE IEEE 802.16 ARCHITECTURE}

The section briefly summarizes the operations of MAC and PHY layers in the IEEE 802.16 standard. Fig. 1 illustrates the architecture of IEEE 802.16. The CS provides any transformation or mapping of external network data, received through the CS service access point (SAP), into MAC service data units (MSDUs) received by the MAC layer through the MAC SAP. This sublayer includes classifying external network SDUs and associating them to the proper MAC service flow identifier
(SFID) and connection ID (CID). It may also include such functions as payload header suppression (PHS).

The MAC CPS provides the core MAC functionality of system access, bandwidth allocation, scheduling, contention mechanism, connection establishment, and connection maintenance. It receives data from various $\mathrm{CSs}$, through the MAC SAP, classified to particular MAC connections. The IEEE 802.16-2004 standard supports four quality-of-service scheduling types: unsolicited grant service (UGS) for the constant bit rate (CBR) service, real-time polling service (rtPS) for the variable bit rate (VBR) service, non-real-time polling service (nrtPS) for non-real-time VBR, and best effort service (BE) for service with no rate or delay requirements. In 802.16e standard, there is an additional service type called extended real-time polling service (ertPS) for voice over IP (VoIP) service with silence suppression.

These quality-of-service (QoS) classes are associated with certain predefined sets of QoS-related service flow parameters, and the MAC scheduler supports the appropriate data handling mechanisms for data transport according to each QoS classes. 
The upper-layer protocol data units (PDUs) are inserted into different level queues with an assigned CID in the MAC layer after the SFID-CID mapping. These data packets in these queues are treated as MSDUs and then will be fragmented or packed into various sizes according to the MAC scheduling operations, and be processed by a selective repeat automatic repeat request (ARQ) block mechanism if the ARQ-enabled function is turned on.

For the UL traffic, each SS should range to the BS before its entering the system. During the initial ranging period, the SS will requests to be served in the DL via the particular burst profile by transmitting its choice of DL interval usage code (DIUC) to the BS. Then the BS will command the SS to use a particular uplink burst profile with the allocated UL interval usage code (UIUC) with the grant of SS in UL-MAP messages. The DL-MAP and UL-MAP contain the channel ID and the MAP information elements (IEs) which describes the PHY specification mapping in the UL and DL respectively. They are based on the different PHY specification, like single carrier (SC), single carrier access (SCa), OFDM, and OFDMA. The burst profile include the DIUC, UIUC, and the typelength-value (TLV) encoded information. The TLV encoded information will notify PHY layer of the modulation type, FEC code type, and encoding parameters. The MAC data payload is packed by these encoding type.

The PHY layer requires equally radio link control (RLC), which is the capability of the PHY layer to transition from one burst profile to another. The RLC begins with the periodical BS broadcasting of the burst profiles which have been chosen for the downlink or the uplink connections. After the initial determination of downlink and uplink burst profiles between the BS and a particular SS, RLC continues to monitor and control the burst profiles. The SS can use the ranging with the RNG-REQ message to request a change in downlink burst profile. The channel measurements report request (REPREQ) message will be used by a BS to request signal-tonoise ratio (SNR) channel measurements reports. The channel measurement report response (REP-RSP) message is used by the SS to respond the channel measurements listed in the received REP-REQ.

The IEEE 802.16 uses the frame-based transmission architecture where the frame length is variable. Each frame is called a superframe and divided into two subframes: the DL subframe and UL subframe. In this paper, we focus the frame structure on OFDMA-PHY in time division duplex (TDD) mode. A DL subframe consists of DL subframe prefix to specify the modulation and coding (in PHY mode), the length of the first DL burst, and the broadcast MAC control messages. For example, the downlink channel descriptor (DCD) and the uplink channel descriptor (UCD). Both of them define the characteristics of the physical channels. The DCD and UCD comprise the detail information of the DL burst profile and the UL burst profile.

Although IEEE 802.16 defines the connection signaling (connection request and response) between SS and BS, it does not define the admission control process. All packets from the application layer are classified by the connection classifier based on CID and are forwarded to the appropriate queue. At

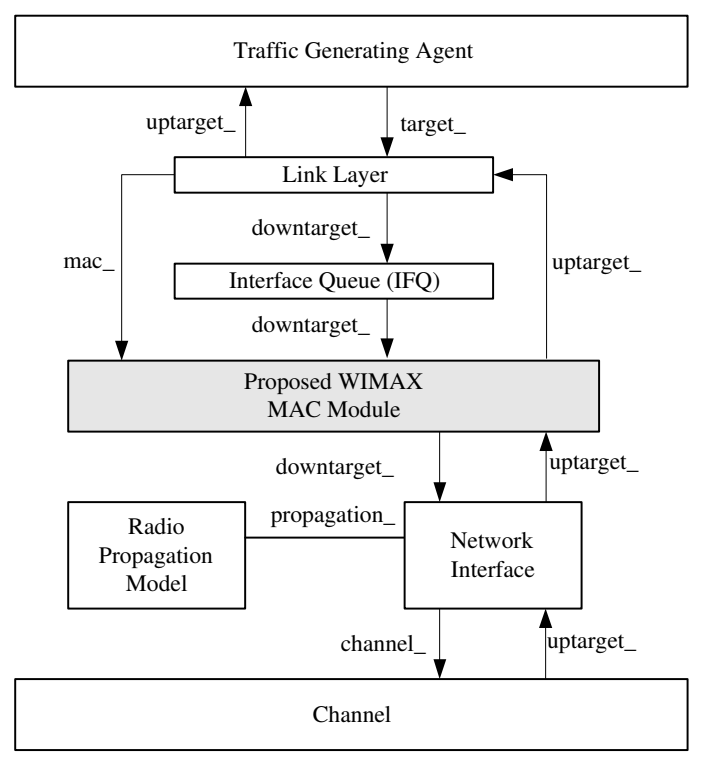

Fig. 2. The relationship between WiMAX object and legacy ns-2 objects.

the SS, the scheduler will retrieve the packets from the queues and transmit them to the network in the appropriate time slots as defined by the UL-MAP sent by the BS. The UL-MAP is determined by the scheduler module based on the BW-request messages that report the current queue size of each connection in SS.

\section{The IEEE 802.16 NS-2 Module}

The developed 802.16-based WiMAX module named as the Mac802_16 class is in accordance with the specifications of the IEEE 802.16-2004 standard [1] and based on the ns-2 version 2.29 [11]. All modules are designed by using objectoriented programming language $\mathrm{C}++$ and modeled as several classes. The relationship of WiMAX module and legacy ns-2 modules, which is based on the original network component stack of the ns-2, is shown in Fig 2. It illustrates the type of objects for the traffic generating agent (TGA), the link layer (LL), the interface queue (IFQ), the designed MAC layer (WiMAX module), and the PHY layer (Channel).

First, the TGA is considered simply an application level traffic generator that generates VoIP, MPEG, FTP, and HTTP traffic, etc. These traffic are classified into five different types of service, the UGS, rtPS, ertPS, nrtPS, and BE, with different priorities. All packets will be transferred to different types of priority queues according to their service types by using CS layer SFID-CID mapping mechanism. The data packets in these queues are treated as MSDUs and will be selected to pass into the WiMAX module in a round robin manner.

While the WiMAX module in the SS receives the MSDUs from Queue object, the MAC management component initiates the ranging process to enter the WiMAX system or transmits the MSDUs according to the scheduled time obtained from UL-MAP. Once the process has been successfully finished in the MAC layer, the Network Interface will add a propagation delay and broadcast in the air interface. The Channel object we use is the WirelessPhy class. 


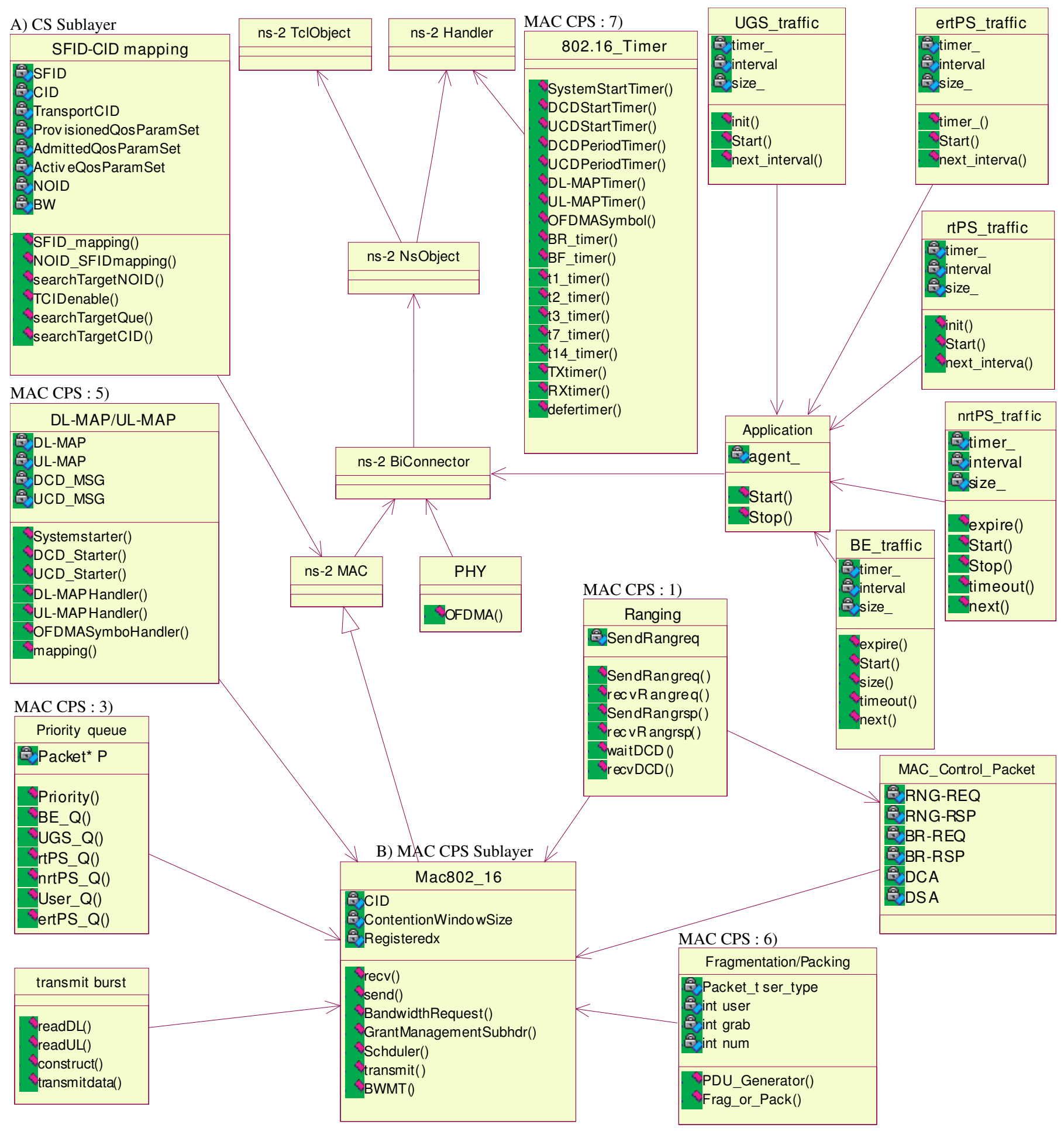

Fig. 3. The class diagram of the designed WiMAX module.

The WiMAX module also receives packets from the air interface passed from another nodes, and it then determines whether the packet is control packet or not. If the packet is a control packet, the MAC management object will take corresponding procedures according to the control packet; otherwise, the packet will be passed to LL object after the defragmentation process. Finally, the TGA will receive the packets from the LL object.
The proposed WiMAX module is composed of the CS sublayer, the MAC CPS sublayer, and the PHY layer. All classes of components are depicted in Fig. 3, which graphically shows these objects, attributes, and their relationships. The BS and SS are identified as its corresponding numbers, which are determined in the OTcl object. The detail components of the WiMAX module are described as following. 


\section{A. The CS Sublayer}

The CS sublayer has two major functions: 1) transforming the IP address (from the upper layer) into several SFIDs or the reverse transformation (from SFID to IP address), and 2) recording the mapping between a SFID and a transport CID (TCID). These functions enable the MAC layer to keep the essential information of the upper layer SDUs about their QoS parameters and destination addresses.

1) IP-SFID mapping: The SDUs which come from the application layer contain their corresponding destination addresses and their service types. An IP-SFID mapping function should record and classify the characteristics of the requesting packets for future IP-MAC mapping. A SFID is used for either the DL transmission with QoS parameters reference or the UL transmission with IP lookup.

2) SFID-TCID mapping: The SFID-TCID mapping is a main function of the CS sublayer for SFID to TCID mapping, which defines the QoS class of the service flow associated with the connection. For the UL traffic, the SS will send a bandwidth request header with the primary CID to the BS for data transmission by invoking the BandwidthRequest () function if it does not obtain a TCID. The SS can add, change, or delete its obtained bandwidth via bandwidth management messages: dynamic service addition, change, and deletion (DSA, DSC, and DSD) later. For the DL traffic (from Internet), the SFID_mapping () function (in the BS) will determine whether the SS obtained a TCID. If not, this function will generate an unused TCID for the SS; otherwise, this function will transfer the MSDU into the corresponding QoS queue.

When SS sends a bandwidth request message to the BS for bandwidth request at first time, the BS will allocate ten SFIDs (five for DL and five for UL traffic) once and one TCID associated to the request for the SS. The length of the SFID and TCID are 32-bit and 16-bit (2m+1-0xFEFE) long, where $m$ is a variable depending on the setting of the operator, respectively. After the mapping operation, the SFIDTCID mapping will be recorded in both sides of the BS and the SS. Moreover, the SS also records the provided QoS service flow in the ProvisionQoSParamSet and the using QoS service flow in the AdmittedQoSParamSet, and the active QoS service flow in the ActiveQoSParamSet.

\section{B. The MAC Sublayer}

The MAC CPS sublayer is the main part of the MAC and maintains the MAC operations and management messages of the system. The management messages such as DCD, UCD, DL-MAP, UL-MAP, DSA, DSC, DSD, RNG-REQ, RNG-RSP, and so forth are generated in this sublayer. The concept of CPS architecture design follows the block diagram shown in Fig. 1. The main body of the MAC CPS is constructed by a Mac802_16 class, which contains several independent functions such as Ranging(), Fragmentation(), and BandwidthRequest(), etc. The detailed functions are described as follows.

1) Ranging: The initial ranging process is the first step in our module for a SS to enter the network. First, a new
SS has to scan for the DL channel and establish synchronization with the BS. After synchronization with the BS, the SS will obtain transmit parameters from the UCD message, which is periodically generated by the BS, to recognize the channel information for transmission. While an unregistered SS receives a packet from the Queue object, it will start the ranging process to notice the BS for entering the system. The SS sends the RNG-REQ management message in the ranging interval which is defined in the UL-MAP issued from the BS with quadrature phase shift keying (QPSK) 1/2 coding rate modulation for contending the entry of the system. The entering process follows the random backoff mechanism with a initial backoff countdown interval of $\left(0, \mathrm{CW}_{\min }-1\right)$ where the $\mathrm{CW}_{\min }$ is the minimum contention window size and is equal to 32. At ranging period, the backoff time is uniformly chosen in the range $\left(0, \mathrm{CW}_{\min }-1\right)$. After each unsuccessful transmission, the $\mathrm{CW}_{\min }$ is doubled up to a maximum value $\mathrm{CW}_{\max }=2^{m} \mathrm{CW}_{\min }$. The $\mathrm{CW}_{\max }$ value is set to 1024 as defined in the standard. The SS uses CID value of zero to send RNG-REQ and starts a timer for waiting the a RNGRSP message from the BS. These processes are operated in two functions rng_req() and rng_rsp().

If the SS receives RNG-RSP before the timer expiration, the ranging process is successful. Otherwise, the SS will select a new backoff window size for a new ranging process. The collision detection of the RNG-REQ is set by the receiver timer. If there are more than one RNG-REQ message is sent within the time interval, these RNG-REQs are treated as collision. Otherwise, this message is successful. When the BS receives a RNG-REQ message from the SS, it will decide to let the SS join the networks or not. After determination, the BS will reply a RNG-RSP message following the DL-MAP among the next several superframes. The RNG-RSP contains a unique basic CID and primary CID to the SS for future communications.

2) MAC management: Five kinds of messages, DCD, UCD, DL-MAP, UL-MAP, and bandwidth request (BR), are used in this function. Each message has its own management message type and they can be discriminated by each other. In the DCD, it includes the management message type, downlink channel ID, TLV encoding information for the overall channel, and the downlink burst profile. The DCD channel encoding is composed of the TLV specific, which includes all the channel information, such as the DL burst profile (may appear more than once), the frame duration, PHY type, power adjustment rule, channel number, the transmit/receive transition gap (TTG) and the receive/transmit transition gap (RTG), the frequency of the downlink center frequency, the BSID, the frame duration code, and the frame number.

The important point of the DCD is the downlink burst profile, which includes the DIUC (in order to map to the DLMAP) and the TLV encoded information. In the TLV encoded information DCD burst profile, there are FEC code type, DIUC mandatory exit threshold, as well as the DIUC minimum entry threshold. The FEC code type can indicate the modulation type of the burst. The DIUC mandatory exit threshold will define the range of the CINR and which indicated DIUC can no longer be used, and where this change to a more robust DIUC 
is required. Similarly, the DIUC minimum entry threshold is the minimum required CINR to start using this DIUC.

In the UCD, it includes the management message type, ranging backoff start, ranging backoff end, request backoff start, request backoff end, and the TLV encoding information for the overall channel. The major of UCD is the TLV encoding information for the overall channel, it constructs the uplink burst profile. The same as the downlink burst profile, the uplink burst profile also contains the FEC code type and modulation type. The ranging data ratio is also included in the uplink burst profile, it means the reducing factor between the power used for this burst and power should be used for CDMA ranging. The last TLV encoding information in uplink burst profile is the normalized $\mathrm{C} / \mathrm{N}$ override. This is a list of numbers, where each number is encoded by one nibble and interpreted as a signed integer. All above-mentioned MAC messages are triggered by specific timers.

First, all traffic flows are generated by the traffic generating agent as shown in Fig. 2. These data flows will be treated as the basic packet object defined in ns-2. Finally, these packets will come to the Mac802_16 through the interface queue (IFQ) and be treated as the MSDUs. Once the MSDU comes, the SFID_Mapping () is invoked in order to classify the MSDUs into several groups, such as UGS, rtPS, ertPS, nrtPS, and BE. If the SFID is set active, then the MSDU will be transferred into the queue labeled as SFID number. Otherwise SFID_Mapping () will assign a TCID and set the corresponding SFID active.

After the service flow classification, these MSDUs will be transferred into their corresponding queue and wait to be served. In this stage, either in DL or UL channel, the BS has to manage the bandwidth by invoking the bandwidth management function BWMT (), which plays the admission control of each SS in UL and the DL bandwidth management.

The SS may request to perform bandwidth request with BS by using BandwidthRequest () and the related parameters, e.g., CID, type, encryption control (EC), and header type (HT), etc. The HT field of the MAC header is set to one for indication of a BR. The SS will calculate its required bandwidth and set the BR field (19 bits) to corresponding bandwidth (1-524287 bytes). Afterward the SS will continuously observe the upcoming UL-MAPs for checking whether its request is successful or not. The bandwidth request process follows the random backoff approach as described in ranging process. Once the requested bandwidth is admitted by the BS, the SS can invoke GrantManagementSubhdr () for future grant management if SS needs more bandwidth. The GrantManagementSubhdr() will generate a subheader for indication of piggyback request, poll-me, or slip indicator of the active TCID.

3) Priority queue: In the BS or SS, the packets come from the upper layer will first be delivered to Priority () . According to the TCID and its service type: UGS(6), $\operatorname{rtPS}(5)$, ertPS(4), nrtPS(3), BE(2), the Priority () will make priority classification. The Priority () generates an exclusive queue to storage these packets based on the SFID. Finally, packets will be treated as MSDUs and segmented into different queue function, e.g., UGS_Q (), rtPS_Q (), etc.
4) Scheduler: The Scheduler() function is in charge of selecting these queued MSDUs according to the admitted bandwidth. The selection police of the scheduler in our designed module is the weighted Round-Robin method. The strategy guarantees that the $\mathrm{BE}$ traffic still obtains a minimum bandwidth for transmission if the traffic load is extremely heavy. The ARQ function is optional in our module.

5) $D L-M A P / U L-M A P$ : The DL-MAP and UL-MAP are periodically generated for announcing the information of the arrangement of the DL and UL periods in the superframe. These two messages are handled by DLmapHandler() and ULmapHandler(). There are management message types, PHY synchronization field, DCD count, base station ID, and DL-MAP_IEs (IE: information element) in the DLMAP message. The important part in the DL-MAP are the DL-MAP_IE()s, which are composed by the type-lengthvalue (TLV) encoding. Each DL-MAP_IE is generated by DL_MAP_IE ( ) and each DL-MAP_IE contains the downlink interval usage code (DIUC), the CID, the number of CIDs assigned for this IE, the OFDMA symbol offset and the subchannel offset, the number of the OFDMA symbols, and the number of the subchannels.

The structure of UL-MAP is similar to the DL-MAP, but the differences between them are the uplink channel ID and the allocation start time. The uplink channel ID is the identifier of the uplink channel to which this message refers. The allocation start time is the effective start time of the uplink allocation defined by the UL-MAP. The UL-MAP_IE structure is also similar to the DL-MAP_IE. The capability of the DL-MAP and the UL-MAP will decide the time domain and the frequency domain in the frame space.

6) Fragmentation/Packing: The packet fragmentation or packing process is executed by PDU_Generator () function. This function grabs the MSDUs from QoS queues (UGS, rtPS, ertPS, nrtPS, and BE) and produces MPDUs depending on the command of the Scheduler(). It will generate the generic MAC header for each data payload. Fragmentation is the process that divides a MSDU into one or more MPDUs. If packing is turned on for a connection, the MAC may pack multiple MSDUs into a single MPDU. In this module, the input MSDU will be fragmented or packed depending on the length of the MPDU. Due to the reason of simplicity, we set the length of each MPDU fixed. Once the fragmentation or packing process is proceed, the corresponding subheader will also be given to each MSDU contained in one MPDU.

After data fragmentation and packing, the scheduler will invoke Transmit_Data () for data transmission. The treatment of the transmission will be various depending on it happened in the SS or BS. In the SS, the scheduler concatenates the MPDUs into one burst transmission according to the arrangement of the UL-MAP. In contrast to SS, the BS concatenates the MPDUs into one burst transmission according to the DL-MAP, which is generated by the scheduler. This function is periodically triggered once in the superframe to decide to transmit burst data to/from DL or UL whether it is in the BS or SS.

The Assembler () is a process function of defragmentation and unpacking. It will read the MAC header to see 
TABLE I

Parameters Used In the MAC AND Physical Layers

\begin{tabular}{ll}
\hline Parameters & Value \\
\hline \hline \multicolumn{1}{c}{ MAC Layer Parameter } & \\
\hline DL/UL ratio & $2: 1$ \\
Number of OFDMA symbol per frame & 49 \\
Number of OFDMA symbol per frame for data & 48 \\
Number of subchannels & 30 \\
CW $_{\text {min }}$ & 32 opps \\
CW max & 1024 opps \\
Ranging opportunity per Frame & 12 OFDMA symbols \\
Max. number of ranging retry & 10 \\
Bandwidth request opportunity per frame & 12 OFDMA symbols \\
Max. number of bandwidth request retry & 10 \\
Initial ranging CID & 0 \\
Basic CID & $1-1000$ \\
Primary CID & $1001-2000$ \\
Transport CIDs and secondary Mgt. CIDs & $2001-65278$ \\
Broadcast CID & 65535 \\
SFID range & $1-4294967295$ \\
\hline \multicolumn{2}{c}{ Physical Layer Parameter } \\
\hline Spectrum & $5.0 \mathrm{GHz}$ \\
Bandwidth & $20 \mathrm{MHz}$ \\
QPSK 1/2 & $4.99 \mathrm{Mbps}$ \\
QPSK 3/4 & $7.48 \mathrm{Mbps}$ \\
16-QAM 1/2 & $9.97 \mathrm{Mbps}$ \\
16-QAM 3/4 & $14.96 \mathrm{Mbps}$ \\
64-QAM 2/3 & $19.95 \mathrm{Mbps}$ \\
64-QAM 3/4 & $22.44 \mathrm{Mbps}$ \\
QPSK 1/2 & $-79 \mathrm{dBm}$ \\
QPSK 3/4 & $-76 \mathrm{dBm}$ \\
16-QAM 1/2 & $-72 \mathrm{dBm}$ \\
16-QAM 3/4 & $-69 \mathrm{dBm}$ \\
64-QAM 2/3 & $-65 \mathrm{dBm}$ \\
64-QAM 3/4 & $-63 \mathrm{dBm}$ \\
\hline
\end{tabular}

whether this MPDU is fragmented or packed. Furthermore, the subheaders information such as FC in MPDU is also read to recover an original MSDU.

7) 802.16 timer class: The 802.16 Timer class inherits the Handler class with three important functions:

- The start () is used to trigger the timer to start.

- The stop() is used to stop the timer if the event happened before expiration.

- The handle () is used to trigger event while time out. These Timer classes play an important role of the system as sequencing of the events between the BS and SSs in WiMAX networks. For instance, each superframe is started from the DL-MAPTimer, and then triggers the following timers, UL-MAPTimer, DCDPeriodTimer, and UCDPeriodTimer iteratively. Another kind of timer is used to count down the given time before the expected time expires or an event is encountered, e.g., the SS starts a RNG-REQ timer to wait for the RNG-RSP from the BS. All timer intervals defined in the WiMAX module are referred to the IEEE 802.16 standard.

\section{Simulation Parameters and Demonstrations}

The system-specific parameters of the IEEE 802.16 MAC protocol we use are shown in Table I and Table II respectively. The simulation environment, shown in Fig. 4, is set one serving BS to 9 SSs concurrently within a $1000 \mathrm{~m} \times 1000 \mathrm{~m}$ square. All SSs are randomly developed around the BS and the modulation versus distance model is based on [4], i.e., the
TABLE II

SYSTEM TIME PARAMETER

\begin{tabular}{ll}
\hline Parameter & Value \\
\hline \hline OFDMA symbol time & $100.84 \mu \mathrm{s}$ \\
OFDMA frame length & $5 \mathrm{~ms}$ \\
DCD/UCD period & $10 \mathrm{sec}$ \\
Ranging interval & $1210.08 \mu \mathrm{s}$ \\
Bandwidth request interval & $1210.08 \mu \mathrm{s}$ \\
TTG & $200 \mu \mathrm{s}$ \\
RTG & $200 \mu \mathrm{s}$ \\
T1-T26 & as defined in IEEE 802.16 \\
\hline
\end{tabular}

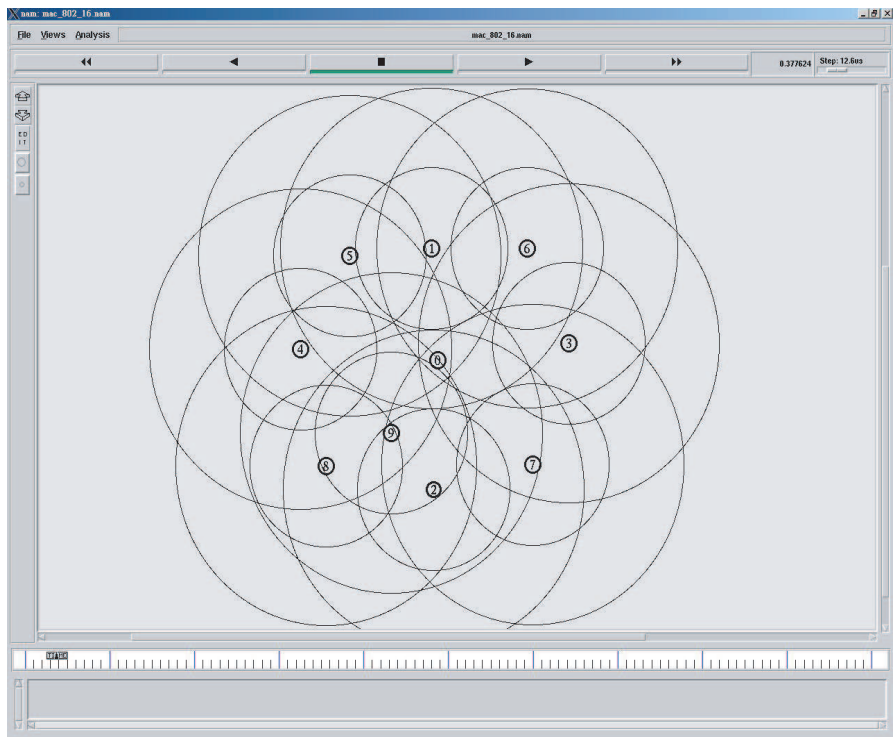

Fig. 4. A snapshot of the simulation topology.

achievable transmission rate depends on how far the distance between the BS and SS is. All SSs execute the ranging request process by adopting QPSK 1/2 encoding rate.

There are three kinds of service flows: UGS, nrtPS, and BE, which are generated from the traffic generating agent, in each SS and the BS. The Internet traffic is treated as the DL traffic to SSs, on the contrary, the UL traffic is the traffic from SSs to Internet. For CBR traffic, there are six UGS connections (three for DL and three for UL) and each connection occupies fixed $1024 \mathrm{Kbps}$ data traffic. For VBR traffic, there are totally 12 nrtPS connections (eight for DL and four for UL) and the data traffic model follows the Poisson distribution with a mean $\lambda=1$ per second and mean data length 192 KBytes (between 128 KBytes-256 KBytes), i.e., each connection occupies about $1.5 \mathrm{Mbps}$. The traffic model of BE is same as VBR traffic model but the numbers of connections are four for DL and two for UL. The priority order of the traffic is UGS, nrtPS, and $\mathrm{BE}$, and scheduled in the weighted round robin manner.

Fig. 5 shows the throughput per each channel. The simulation time persists for 20 seconds. All traffic data are continuously generated throughout the simulation time. We can see that the curve of the obtained throughput of the system oscillates highly since each SS spends some time in dealing with the initial ranging process and the bandwidth request process, which will follow the random backoff mechanism with a initial backoff countdown interval and stand on the 


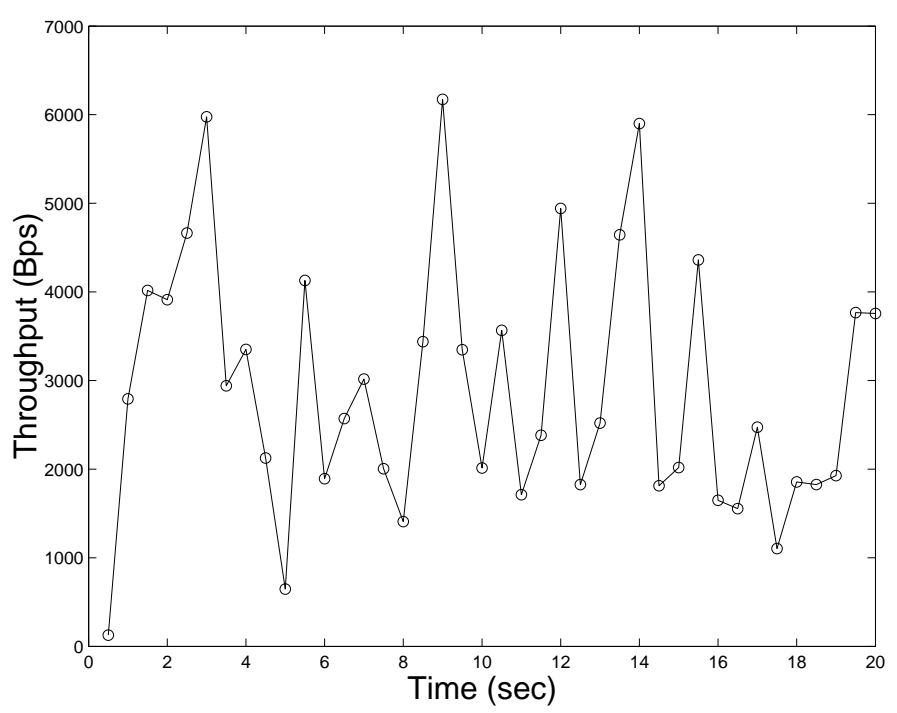

Fig. 5. The channel throughput derived by IEEE $802.16 \mathrm{~ns}-2$ in $20 \mathrm{sec}$ time.

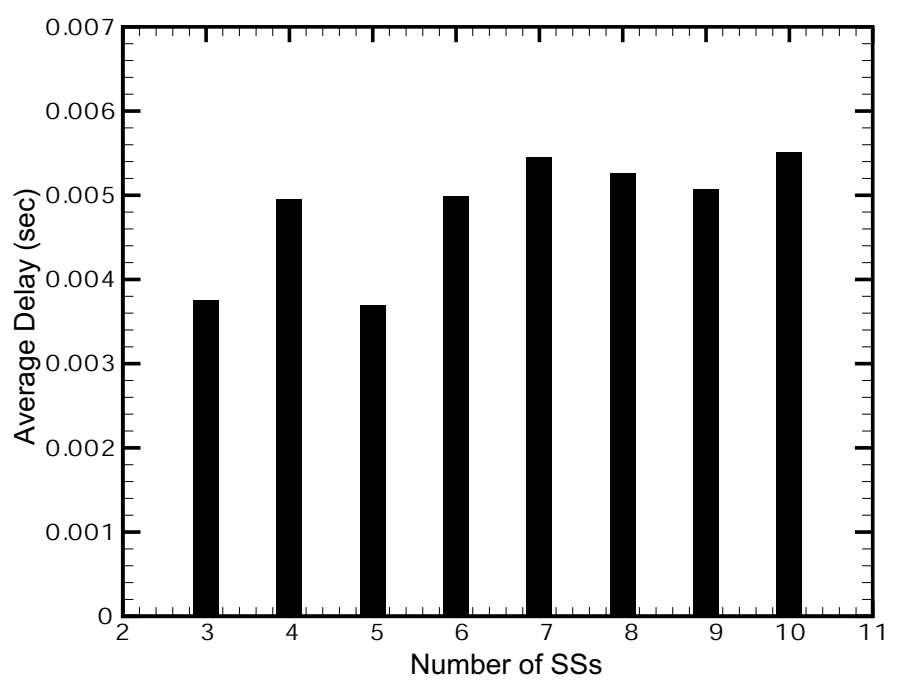

Fig. 6. The average delay ratio versus different number of SSs under the maximum transmission power of IEEE $802.16 \mathrm{~ns}-2$.

random backoff approach. It also spends time on waiting transmit and receiving two ways transition gap (TTG and RTG). Therefore, the curve of throughput oscillates between 127 to 6173 Bytes/sec in the general condition.

Fig. 6 illustrates the average MAC delay under given number of SSs in detail. We can realize that the delay time will increasing with the growth of the number of SSs. The MAC delay are result from the collision of initial ranging and the bandwidth request. After the bandwidth request, the delay will become a balance since the BS will arrange the transmission time in the simulation. The scheduling of different traffic service types mainly will affect the MAC delay because of limited spectral resource. Using the weighted round-robin to access the priority queues is not a better way to schedule the transmission and can be replace to a better scheduler in the future work.

\section{CONClusions}

The paper presents a detailed design and implementation of the WiMAX simulation module for ns-2. The designed module is based on the IEEE 802.16-2004 standard and the legacy ns-2 version 2.29. The unified modeling language (UML) is also adopted to design and analysis the WiMAX module. This module includes a basic point-to-multipoint (PMP) IEEE 802.16 function, a different service flows generator, a simple bandwidth management component, and the scheduler. We also demonstrate a simulation scenario to verify the designed module. We hope that this preliminary WiMAX module can benefit academic researchers and industrial developers for early verification of designing the WiMAX system.

The future work will focus on providing mobility functions [3] of the SS and the relay station (RS). In addition, an efficient bandwidth management algorithm and scheduler algorithm for QoS control are crucial for performance enhancement of the WiMAX system.

\section{REFERENCES}

[1] IEEE 802.16 Working Group, "IEEE Standard for Local and Metropolitan Area Networks-Part 16: Air Interface for Fixed Broadband Wireless Access Systems," IEEE Std. 802.16-2004, Oct. 2004.

[2] --, "Part 16: Air Interface for Fixed Broadband Wireless Access Systems-Amendment 2: Medium Access Control Modifications and Additional Physical Layer Specifications for 2-11 GHz," IEEE Std. 802.16a, Apr. 2003.

[3] --, "Part 16: Air Interface for Fixed Broadband Wireless Access Systems-Amendment 2: Physical and Medium Access Control Layers for Combined Fixed and Mobile Operation in Licensed Bands," IEEE Std. 802.16e, Dec. 2005

[4] Jenhui Chen and Wei-Kuang Tan, "Predictive Dynamic Channel Allocation Scheme for Improving Power Saving and Mobility in BWA Networks," ACM/Springer Mobile Networks and Applications (MONET), 2006.

[5] C. Eklund et al., "IEEE Standard 802.16: A Technical Overview of the WirelessMAN Air Interface for Broadband Wireless Access," IEEE Commun. Mag., vol. 40, no. 6, pp. 98-107, June 2002.

[6] B. Fong, N. Ansari, A.C.M. Fong, G.Y. Hong, and P.B. Rapajic, "On the Scalability of Fixed Broadband Wireless Access Network Deployment," IEEE Commun. Mag., vol. 42, no. 9, pp. 12-1, Sept. 2004.

[7] I. Koffman and V. Roman, "Broadband Wireless Access Solutions Based on OFDM Access in IEEE 802.16," IEEE Commun. Mag., vol. 40, no. 4, pp. 96-103, Apr. 2002.

[8] T. Kwon, et al., "Design and Implementtation of a Simulator Based on a Cross-Layer Protocol between MAC and PHY Layers in a WiBro Compatible IEEE 802.16e OFDMA System," IEEE Commun. Mag., vol. 43, no. 12, pp. 136-146, Dec. 2005.

[9] H. Minn, V.K. Bhargava, K.B. Letaief, "A Robust Timing and Frequency Synchronization for OFDM Systems," IEEE Trans. Wirel. Commun., vol. 2, no. 4, pp. 822-839, Jul. 2003.

[10] K. Wongthavarawat and A. Ganz, "Packet Scheduling for QoS Support in IEEE 802.16 Broadband Wireless Access Systems," Int. J. Commun. Syst., vol. 16, no. 1, pp. 81-96, Feb. 2003.

[11] The network simulator-ns-2. http://www.isi.edu/nsnam/ns/. Page accessed on April 1, 2005. 